\title{
Komplementärmedizin
}

\section{Vitamine und Spurenelemente sind besonders beliebt}

\author{
„Was leistet die Komplementärmedizin als unterstüt- \\ zende Behandlung für Krebspatienten?" fragten wir in \\ MMW Nr. 4/2010, S. 18. Dazu ein Leser:
}

- Was kann die Komplementärmedizin? Das kommt darauf an, wen Sie fragen. Auch unter Komplementärmedizinern ist die Stellung der einzelnen komplementärmedizinischen Verfahren sehr umstritten. Ein Anhänger der Traditionellen Chinesischen Medizin (TCM) schwört auf chinesische Phytotherapeutika, ein Anhänger der orthomolekularen Medizin hingegen wird darauf hinweisen, dass alle Nährstoffe und Spurenelemente im richtigen Verhältnis supplementiert werden müssen. Der Organotherapeut hält all das für Unsinn und schwört auf die karzinotoxische Wirkung von Rinderhirn, Schweinemilz und Schweineplazenta, für wieder andere zählt nur ozonisiertes Eigenblut, Colon-Hydro-Therapie oder Bioresonanztherapie.

Nach eigenen Untersuchungen aus der versicherungsmedizinischen Tagesarbeit (Diagnosis and treatment methods of alternative medicine in private health insurance a quantitative analysis, Versicherungsmedizin. 2008 Mar 1;60(1):27-31) werden komplementärmedizinisch am häufigsten Vitamine, Spurenelemente und Nahrungsergänzungsmittel eingesetzt. Insbesondere wird sehr häufig eine Vitamin-C-Hochdosistherapie als Infusion mit bis zu 3000 mg Ascorbinsäure pro Tag gegeben.

Viele dieser Präparate haben den Status eines Nahrungsergänzungsmittels. Diese gehören juristisch gesehen zu den Lebensmitteln und bedürfen somit keiner Zulassung. Krankheitsbezogene Aussagen und die Aufstellung von Indikationen sind - wie für andere Lebensmittel auch - gesetzlich unzulässig. Trotzdem wird für Nahrungsergänzungsmittel aber intensiv mit Wirksamkeitsversprechen geworben. Propagierte Indikationen für Nahrungsergänzungsmittel sind nicht nur Krebserkrankungen, sondern auch Allergien aller Art, Arthrosen, Asthma bronchiale, KHK, AVK, rheumatische Erkrankungen, Verlangsamung des Alterungsprozesses und Unterstützung der Gewichtsabnahme - um nur einige wenige Anwendungsgebiete zu nennen.

Erstaunlicherweise sind viele Menschen bereit, mehrere hundert Euro pro Jahr - oft sogar mehrere tausend Euro für Nahrungsergänzungsmittel auszugeben. Hauptsache, es dient der Gesundheit!

- Dr. med. Rainer Hakimi, Leitender Gesellschaftsarzt Hallesche Krankenversicherung a. G., Reinsburgstraße 10, D-70178 Stuttgart 